\title{
Realistic wind farm design layout optimization with different wind turbines types
}

\author{
Naima Charhouni ${ }^{1}$ [D $\cdot$ Mohammed Sallaou $^{1} \cdot$ Khalifa Mansouri $^{2}$
}

Received: 11 December 2018 / Accepted: 16 April 2019 / Published online: 25 April 2019

(c) The Author(s) 2019

\begin{abstract}
Seeking for an appropriate design of wind farm (WF) layout constitutes a complex task in a wind energy project. An optimization approach is seriously needed to deal with this complexity, especially with current trend of large WFs area with important number of wind turbines (WTs). The present paper investigates optimization study of realistic offshore WF design layout (horns-rev1). The main objective of the current study is to design WF area that maximizes the extraction of wind power with low cost. In the first step, an optimization model using genetic algorithm with continuous layout representation is developed to look for the optimal design as a function of WTs placement. The effectiveness of such a methodology is validated and compared with the reference and irregular layout of hors-rev1 offshore WF. With the aim to analyze the impact of WTs types on WF objectives, four commercial WTs are considered in the second step. The results showed that designing WF with big WTs gives best design layout. In addition, it demonstrated that selecting WTs based uniquely on rotor diameter size is not always a good idea. It should includes as well the number of WTs that influence significantly the power production and WF cost.
\end{abstract}

Keywords Wind energy project · Design · Wind farm layout · Wind turbines placement · Wake effect · Optimization . Genetic algorithm

\begin{tabular}{|c|c|c|c|}
\hline \multicolumn{2}{|c|}{ Abbreviations } & $\theta$ & Wind direction $\left(^{\circ}\right)$ \\
\hline$x$ & Wind turbines coordinate in $\mathrm{x}$-axis $(\mathrm{m})$ & $C_{\mathrm{p}}$ & Power coefficient \\
\hline$y$ & Wind turbines coordinate in $y$-axis (m) & WTs & Wind turbines \\
\hline \multirow{2}{*}{$d_{\mathrm{su}}$} & Downstream distance between two wind tur- & WF & Wind farm \\
\hline & bines $(\mathrm{m})$ & WFDLO & Wind farm design layout optimization \\
\hline \multirow[t]{2}{*}{$d_{\text {per }}$} & Perpendicular distance between two wind & $\mathrm{COE}$ & Cost of energy \\
\hline & turbines $(\mathrm{m})$ & $\eta_{\mathrm{m}}$ & Gearbox mechanical efficiency \\
\hline$D$ & Rotor diameter (m) & $\eta_{\mathrm{g}}$ & Generator efficiency \\
\hline$R$ & Rotor radius (m) & $D_{\mathrm{wk}}$ & Wake diameter $(\mathrm{m})$ \\
\hline$N$ & Total number of wind turbines & $C_{\mathrm{t}}$ & Trust coefficient \\
\hline$N_{\text {up }}$ & Number of upstream wind turbines & $p$ & Air intensity $\left(\mathrm{kg} / \mathrm{m}^{3}\right)$ \\
\hline$z_{\mathrm{h}}$ & Height of wind turbines (m) & $\alpha$ & Wake decay coefficient \\
\hline$V_{\mathrm{f}}$ & Free incoming wind speed $(\mathrm{m} / \mathrm{s})$ & $z_{0}$ & Surface roughness length $(\mathrm{m})$ \\
\hline \multirow[t]{2}{*}{$V_{\mathrm{df}}$} & Wind velocity deficit $(\mathrm{m} / \mathrm{s})$ & $a$ & Induction factor \\
\hline & & $A$ & Swept area of wind turbines $\left(\mathrm{m}^{2}\right)$ \\
\hline & & $A_{\mathrm{ov}}$ & Overlap area $\left(\mathrm{m}^{2}\right)$ \\
\hline \multirow[t]{2}{*}{$\triangle$} & \multirow{2}{*}{$\begin{array}{l}\text { Naima Charhouni } \\
\text { charhouninaima@gmail.com }\end{array}$} & $C_{\mathrm{WF}}$ & Wind farm cost $(\$)$ \\
\hline & & $P_{\mathrm{WT}}$ & Power produced by wind turbine (MW) \\
\hline \multirow[t]{3}{*}{1} & \multirow{3}{*}{$\begin{array}{l}\text { Department of Mechanics and Integrated Engineering (M2I), } \\
\text { Moulay Ismail University, ENSAM, B.P. 15290, Meknes, } \\
\text { Morocco }\end{array}$} & $P_{\mathrm{WF}}$ & Power produced by wind farm (MW) \\
\hline & & $\eta_{\mathrm{WF}}$ & Wind farm efficiency (\%) \\
\hline & & $\mathrm{CF}$ & Capacity factor of wind farm (\%) \\
\hline \multirow[t]{2}{*}{2} & \multirow{2}{*}{$\begin{array}{l}\text { Department of Signals, Distributed Systems and Artificial } \\
\text { Intelligence (SSDIA), ENSET Mohammedia, Hassan II } \\
\text { University, Casablanca, Morocco }\end{array}$} & $P_{\mathrm{r}}$ & Rated power of wind turbines (MW) \\
\hline & & $V_{\mathrm{r}}$ & Rated wind speed of wind turbines $(\mathrm{m} / \mathrm{s})$ \\
\hline
\end{tabular}




\section{Introduction}

The important interest and efforts devoted by government, energy production, industries and academic research to electricity production from renewable and clean energy with the maturity of existed technologies justify the biggest exploitation of wind energy over the recent years. For instance, the wind energy investments in Europe has received the biggest part of new renewable energy finance about $63 \%$ in 2018, up from 52\% in 2017 [1]. As a result of this evolution, the design process becomes highly complex from the point of the significant energy capacity to be produced, which generally requires hundreds of wind turbines (WTs) to be installed over a limited area. At this stage, several conflicting objectives, mechanisms and constraints have been imposed while requiring a balance between them in order to ensure the viability of wind energy project.

Designing wind farm (WF) layout refers to find the optimal placement of each individual WT from others inside the specific WF boundaries. However, the quality and quantity of expected power produced by WF are generally reduced by various sources of losses in which wake effect is considered the most influencing one [2]. Many published works provide that wake interferences generated randomly by upstream turbines cause a significant reduction of wind speed and increase turbulence intensity [3,4]. Consequently, it gives rise to maintenance cost and diminishes the power production of downstream turbines. In this regard and in order to mitigate the wake effect among each WTs placement, wind farm design layout optimization (WFDLO) remains the best strategy to maximize WF performances.

Up to now, this problem has received a big attention by scientific research which is justified by the huge numbers of studies that have been addressed in the literature. The main objective conducted by these works is developing numerical algorithms that maximize power/energy production (in other words decreases the wake effect) and minimize the cost while satisfying certain constraints. These algorithms are based on different optimization techniques in which heuristic methods widely used. These methods includes genetic algorithm [5, 6], evolutionary algorithm [7], particle swarm optimization algorithm [8] and among others methods that can found in detail in these references $[2,9,10]$.

Before reviewing some works that have done in this context, it is important to note that there are two basic approaches to represent the WF layout using heuristic methods $[11,12]$. The first proposed approach consists of discretizing WF area into equal square grids. The center of each square represents the possible placement of WTs indicated by binary variables ( 0 and 1$)$. Whilst the second approach called the continuous representation assumed that WTs can be placed at any position within the specified WF boundaries. In the literature, discrete approach is widely used to describe WF layout. But using such a methodology limits the feasible solutions of WTs placement due to the predefined grids. Thus, using continuous approach sound more appropriate to explore all feasible positions of wind turbines within wind farm area.

Since the two works conducted by Mosetti et al. [13] and Grady et al. [14] to optimize WFDL using genetic algorithm with discrete approach, much studies have been developed. They focus on improving WF performances through several variables and using various optimization methods as mentioned before. The vast majority of studies are interested to optimize the cost per unit of power (COE) using turbines with different hub height. For instance, Chen et al. [15] proposed an optimization approach based on greedy algorithm to investigate the benefit of using WTs with different hub height $(78 \mathrm{~m}$ and $50 \mathrm{~m}$ ) rather than identical hub heights $(78 \mathrm{~m})$ considering the same cases study of Mosetti et al. [13]. The results obtained show a significant improvement in COE value. Note that this study gives importance only to the concept of using WTs with different hub height neglecting the sensitivity variation of wind speed and wake interactions with tower height. This aspect is then studied by MirHassani et al. [16] who developed a mathematical programming model using linearization technique and iterative method to evaluate power production under various conditions of wind speeds and directions. The numeral results showed better arguments compared with the results found in References [13, 15].

Other research studies attempted to investigate the influence of rotor diameter on the COE. Mustakerov and Borissova [17] optimize WTs placement for both square and rectangular area using combinatorial algorithm. In their study, they strived to optimize COE under two wind direction cases (uniform and predominant) as function of type and number of WTs. They concluded that installing WTs with big size is more beneficial than WTs with small size. This study suffers from the lack of appropriate modeling of wake effect and WF cost that have to be assumed as function of WTs size and number. Chowdhury et al. [18] confirmed that employing different diameters (case 2) with assumption of using uniform WT type for entire WF can improve the power output. They solved the problem using mixed discrete PSO algorithm. Meanwhile, in their last case study they showed that choosing different WTs type instead of identical ones to be installed in WF can enhance the capacity factor by $2 \%$. In the same manner, Rahbari et al. [19] combine the quadratic assignment problem with genetic algorithm to found the optimal 
arrangement of WTs. They also proved that using different types of WTs achieved a high WF efficiency.

According to the reviewed studies, we remark that the vast majority focuses on numerical approach without considering the appropriate modeling of some basic functions of WF. For example, a large part of developed works optimize COE using the simplified cost model proposed by Mosetti et al. [13].This later is assumed to be a function only of WTs number, which cannot represent the variation of COE with changing hub height or rotor diameter. In addition, a few works tacked the optimization problem considering the impact of rotor diameter size and estimate WF cost as a function of this crucial design variable but unfortunately cannot be exploited furthermore. For these reasons, we aim to contribute in the present study to investigate new optimization of WF design layout including these following points:

- Considering WTs types (rotor diameter), number and placement choice as variables of optimization.

- The cost of WF is estimated as a function of WT design variables to be more realistic.

- The power production is optimized using genetic algorithm with continuous approach allowing the exploration of unlimited WF layouts.

- The optimization study is based on real offshore WF Horns Rev1 obtained from the literature [20, 21].

Thereafter, the paper continues with presenting the basic models for WF power and cost calculation in "Wind farm modeling". The optimization procedure and case study are, respectively, explained in detail in "Optimization methodology" and "Description of case study". "Optimization results and discussions" discussed the optimization results of this current study. The last section summarized the conclusions and possible future research studies.

\section{Wind farm modeling}

\section{Wake modeling}

Wind speed passing through upstream WTs is modified by the phenomenon called wake effect. The growth of this effect is characterized by reduced wind speed and increased turbulence intensity in downwind region. Indeed WTs placed in wake region produce less energy and require high maintenance cost as compared with upstream WTs. Thus, modeling appropriately wake effect that plays a key role in determining WTs placement is a serious need to take into account during the WFDLO.

Until now, several wake models have been developed describing the proprieties of wind velocity deficit $[22,23]$. They can be classified into two categories: computational and analytical wake models. The computational wake models based on solving the Navier-Stokes equation are generally considered more accurate but require high computational cost that makes their uses in WFDLO impossible [24]. Thereby, analytical wake models that characterize the far wake effect and based on analytical solution of wind speed velocity are highly recommended within optimization process, especially for large WF with important WTs number $[25,26]$. One of the most widely used is Jensen wake model [27] with linear assumption of wake expansion as shown in Fig. 1. This can be probably attributed to their capacity to predict the power losses with certain accuracy compared to other analytical models, as reported in Ref. [25]. Thus, this model is used in the current study to compute the reduction of incoming wind speed. According to Jensen wake model, the $V_{\mathrm{df}}$ is expressed as follows:

$V_{\mathrm{df}}=V_{\mathrm{f}}\left(\left(1-\sqrt{1-C_{\mathrm{t}}}\right)\left(\frac{D}{D_{\mathrm{wk}}}\right)^{2}\right)$,

where $D_{\text {wk }}$ represents the wake diameter formulated by:

$D_{\mathrm{wk}}=D+2 \alpha s_{\mathrm{sw}}$.

To describe and optimize WF layout, WTs positions are assigned by Cartesian coordinates $(x, y)$ as follows:

$$
\operatorname{Pos}\left(\left[\begin{array}{c}
\mathrm{WT}_{1} \\
\cdot \\
\cdot \\
\mathrm{WT}_{N}
\end{array}\right]\right)=\left[\begin{array}{c}
x_{1}, y_{1} \\
\cdot \\
\cdot \\
\cdot \\
x_{N}, y_{N}
\end{array}\right] \text {. }
$$

The distances between upstream turbines $i$ and downstream one $j$ are calculated as follows:

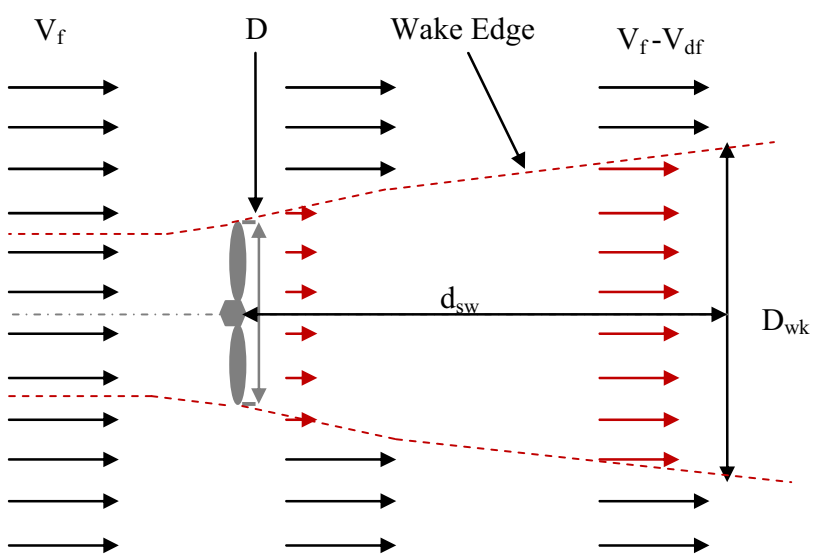

Fig. 1 Illustration of Jensen wake model [27] 
$\left\{\begin{array}{l}d_{\mathrm{sw}}=\left(x_{i}-x_{j}\right) \cos (\theta)-\left(y_{i}-y_{j}\right) \sin (\theta) \\ d_{\mathrm{per}}=\left(x_{i}-x_{j}\right) \sin (\theta)+\left(y_{i}-y_{j}\right) \cos (\theta)\end{array}\right.$.

The wake decay and trust coefficients are considered constant, respectively, in this study but they can be calculated as follows [16]:

$\alpha=\frac{0.5}{\ln \left(\frac{z_{\mathrm{h}}}{z_{0}}\right)}$,

$C_{\mathrm{t}}=4 a(1-a)$.

It is well known that WF composed by an important number of WTs operated all at the same time. In this case, downstream turbines can be influenced not only by one WT wake but also by multiple interferences generated among several upstream turbines. Hence, the total wind velocity deficit for certain WTs can be determined by [28]

$V_{\mathrm{dft}}=\sqrt{\sum_{i=1}^{N_{\mathrm{up}}}\left(\frac{A_{\mathrm{ov}}}{A}\right)\left(V_{\mathrm{df}}\right)^{2}}$,

where $A_{\text {ov }}$ is the overlap area between downstream turbine area and wake region. It reflects the level of wake interaction between several WTs. In general, there are two interaction levels: partial interaction in which downstream turbines are partially operated within wake region generated by upstream WTs, and total interaction where downstream turbines are fully affected. Determining the $A_{\mathrm{ov}}$ is based on some conditions that have to be satisfied.

- For partial wake interactions, this inequality is verified:

$$
R_{\mathrm{wk}}-R<d_{\mathrm{per}} \leq R_{\mathrm{wk}}+R .
$$

And the overlap area can be formulated as [28]:

$A_{\mathrm{ov}}=R_{\mathrm{wk}}^{2} \cos ^{-1}(F 1)-R^{2} \cos ^{-1}(F 2)+\frac{1}{2} \sqrt{F 3}$,

$$
\left\{\begin{aligned}
F 1= & \frac{d_{\mathrm{per}}^{2}-R^{2}+R_{\mathrm{wk}}^{2}}{2 R_{\mathrm{wk}} d_{\mathrm{per}}} \\
F 1= & \frac{d_{\mathrm{per}}^{2}+R^{2}-R_{\mathrm{wk}}^{2}}{2 R_{\mathrm{wk}} d_{\mathrm{per}}} \\
F 1= & \left(d_{\mathrm{per}}-R+R_{\mathrm{wk}}\right)\left(d_{\mathrm{per}}+R-R_{\mathrm{wk}}\right)\left(-d_{\mathrm{per}}+R+R_{\mathrm{wk}}\right) \\
& \left(d_{\mathrm{per}}+R+R_{\mathrm{wk}}\right)
\end{aligned}\right.
$$

$$
R_{\mathrm{wk}}-R \geq d_{\mathrm{per}}
$$

And the overlap zone can be written as:

$A_{\mathrm{ov}}=\frac{\pi D^{2}}{4}$

In the case of no wake interaction, the following inequality is satisfied:

$d_{\mathrm{per}}>R+R_{\mathrm{wk}}$

$A_{\mathrm{ov}}=0$,

According to expressions above, it is clearly shown that wind velocity reduction is related principally to crucial variables written below:

- Incoming wind speed $V_{\mathrm{f}}$.

- Wind direction.

- Trust coefficient $C_{\mathrm{t}}$.

- Wake decay coefficient $\alpha$ (in other words).

- Inter-space between turbines $d_{\mathrm{sw}}$ and $d_{\text {per }}$.

- Rotor diameter of WT $D$.

- Number of WTs $N$.

\section{Power production modeling}

To estimate the power production of WF under wake effect, we need at the first stage to determine the power generated by each individual WT. There are many expressions to approximate the power curve of WT that are elaborated in detail by Carrillo et al. [29]. Thus, the power production of WT is estimated as follows:

$P_{\mathrm{WT}}=\frac{1}{2} \rho \pi \frac{D^{2}}{4} C_{\mathrm{EF}}\left(V_{\mathrm{f}}-V_{\mathrm{df}}\right)^{3}$

where $C_{\mathrm{EF}}$ represent the efficiency factor expressed as follows:

$C_{\mathrm{EF}}=C_{\mathrm{P}} \eta_{\mathrm{m}} \eta_{\mathrm{g}}$

In the present study, $C_{\mathrm{EF}}$ is assumed to be equal to $40 \%$.

The total power generated by WTs operating under wake effect is

$P_{\mathrm{WF}}=\sum_{i=1}^{N} P_{\mathrm{WT}}$

Wind farm efficiency is obtained using this equation:

$\eta_{\mathrm{WF}}=\frac{P_{\mathrm{WF}}}{N\left(\frac{1}{2} \rho \pi \frac{D^{2}}{4} C_{\mathrm{EF}} V_{\mathrm{f}}^{3}\right)}$.

- For total interaction level, this inequality is verified: 


\section{Cost modeling}

The main goal during the development of any power project is to generate energy at the lowest possible cost. At scientific level, the cost modeling is one of the most complicated issues in WF design project. The majority of works published in the stage of WFDLO used the simplistic normalized model introduced by Mosetti et al. [13]. It assumes that WF cost is only a function of WTs number. The uses of such model to estimate WF cost with different WT types cannot give correct results because it does not include the different features related to a WTs design such as rotor diameter, hub high and rated power. To predict WF cost more accurately and as function of these design features, we propose in this study to relate the cost of WF to WT cost. This assumption can be justified by the fact that WT cost constitutes the most expensive component in WF and takes large part of wind energy finance with about $44 \%$ for onshore farms and $64 \%$ for offshore farms [10]. Therefore, we use in this study the cost model proposed in reference [2] that consists of the sum of rotor-nacelle assembly (RNA) cost as a function of rated power and the cost of WT tower as a function of WT design variables. Accordingly, the WF cost can be written as follows:

$C_{\mathrm{WF}}=N\left(1170 \mathrm{P}_{\mathrm{r}}+\left(1.5\left(0.016 D^{2.8}\left(\frac{z_{\mathrm{h}}}{D}\right)^{1.7}\left(\frac{P_{\mathrm{r}}}{A}\right)^{0.6}\right)\right)\right)$.

\section{Optimization methodology}

Any optimization problem including WF design layout requires the definition of four essential elements: the design variables, the constraints, the objective function and optimization method. The formulation of these elements in the context of WF design layout is explained in subsections.

Design variables They consist of input parameters that can be varied to find the optimum solution. In our optimization study, consider these following design variables: position of each WT $(x, y)$, WT types (diameter rotor) and number of WTs.

Constraints They describe the variation fields of design variables which mean that all solutions are not feasible due to the defined restrictions. Two basic constraints are respected during the optimization of WF layout. The first constraint represents the limit boundary of WF outside of which WT cannot be placed. This constraint is expressed as follows:

$\left\{\begin{array}{l}x_{\min } \leq x_{i} \leq x_{\max } \\ y_{\text {min }} \leq y_{i} \leq y_{\text {max }}\end{array} \forall i=1,2, \ldots, N\right.$, where $x_{\min }, y_{\min }, x_{\max }$ and $y_{\max }$ represent the minimum and maximum boundaries of WF area

The second constraint defines the maximum inter-space required between two WTs. In our study, it is assumed to be equal to five times the rotor diameter and can be formulated by the following equation:

$\sqrt{\left(x_{i}-x_{j}\right)^{2}+\left(y_{i}-y_{j}\right)^{2}} \leq 5 D$.

Objective function The objective function is the criteria to be optimized (maximized or minimized). Our main aim is to maximize the power production of WF under the presence of wake effect. The objective function denoted by FOBJ is written as:

$\mathrm{FOBJ}=\max \left(P_{\mathrm{WF}}\right)$.

It worth to mention that in the current study, other criteria such as efficiency (Eq. 18) and capacity factor (Eq. 23) are evaluated:

$\mathrm{CF}=\frac{P_{\mathrm{WF}}}{\text { Power capacity }}$,

where power capacity is equal to $160 \mathrm{MW}$.

Optimization method In broad sense, optimization method is strongly dependent on the nature of optimization problem. It is well known that due to the non-linear behavior of power production that has to be evaluated under various types of variables (design/natural) WF design optimization cannot be solved by traditional approach such as trial and error or deterministic methods. Thence evolutionary algorithms are widely used in a particular genetic algorithm (GA) [2, 9]. This can be justified by its ability to handle complex and non-linear problems. Accordingly, we are interested in our study to use this method of optimization based on continuous layout approach in which WTs can be located at any position within WF area.

Figure 2 illustrates the flowchart of optimization procedure followed to find the optimal layout of WF. Once the input data are sited and aforementioned models are programmed in Matlab@ software, optimization process began to seek for optimal solution. The GA search procedure consists of the main following steps:

- Step 1: Creation of initial population GA began with generating a random population of $\mathrm{n}$ WTs position with respect to the two aforementioned constraints.

- Step 2: Evaluation of objective function WF layouts resulted from previous phase is done through objective function (Eq. 22)

- Step 3: GA operators The first operator started to select individuals that will contribute in the next population generation. The selection probability permits selecting the best individuals from the current generation. Cross- 


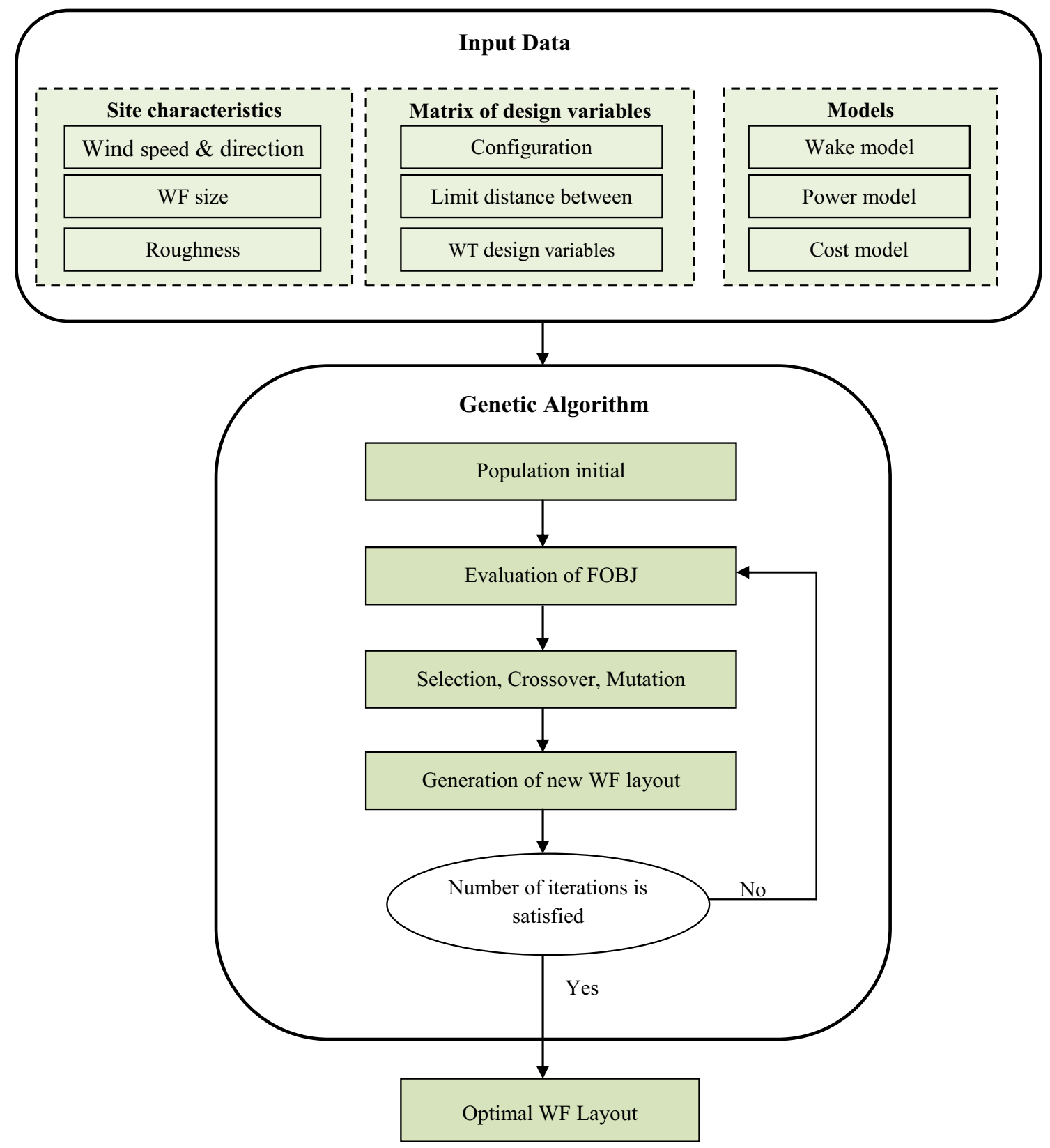

Fig. 2 Flowchart of WFDLO using genetic algorithm

over operator aim is to exchange two couples of genes (position) to produce new design layout. As crossover function, mutation operator is activated randomly with a certain probability (which is a parameter of GA) to modify one gene (position) of individual to another random and allowable position.

- Step 4: Generation of new wind farm layout The population of WF layouts that results from applying GA operators produces new design solution that will replace the old population. All steps above are repeated to ensure the generation of new population of feasible design layout at each iteration of optimization process until reaching the maximum number of iterations. Genetic algorithm parameters used in our study are summarized in Table 1.

\section{Description of case study}

As explained in previous sections, WF design layout is influenced by various factors, such as inter-space between turbines, way of sitting turbines, type and number of WTs 
Table 1 Defined parameters of genetic algorithm

\begin{tabular}{ll}
\hline Parameters & Values \\
\hline Size of initial population & 150 \\
Selection pressure & 3 \\
Crossover probability & 0.25 \\
Mutation probability & 0.75 \\
Iterations number & 400 \\
\hline
\end{tabular}

Table 2 Specific information of wind farm site and wake parameters

\begin{tabular}{ll}
\hline Site roughness & 0.001 \\
Mean wind speed & 10.6 \\
Wind direction & 0 \\
Wake decay coefficient & 0.04 \\
Air density & 1.225 \\
\hline
\end{tabular}

among other factors that impact directly the power production and WF cost. To better understand the influence of these factors on design solutions, we strive in the present study to analyze and compare several scenarios of WFDLO. The study is conducted based on real offshore WF Horns Rev available in literature [20, 21]. The Horns Rev offshore WF is located in the North Sea $14 \mathrm{~km}$ from the west coast of Denmark and extends approximately over $20 \mathrm{~km}^{2}$. It consists of total rated power capacity equal to $160 \mathrm{MW}$ and of 80 Vestas WTs with rotor diameter of 80 and rated power of $2 \mathrm{MW}$. WTs are ranked in regular layout of 8 rows by 10 columns with an inter-distance of $7 D$ among the dominant wind direction $\left(0^{\circ}\right)$. The specific information related to Horns Rev offshore WF is given in detail in Table 2.

It is worth to keep in mind that in the present study we investigate and compare different design layouts of Horns Rev offshore WF considering two principal case studies described below.

\section{Case 1: comparison of three design layout of Horns Rev offshore wind farm}

In this case, we considered three different design layouts of Horns Rev offshore WF: reference layout (which is regular), irregular layout and optimized layout. The three layouts are compared in terms of power production, capacity factor and WF efficiency considering only the WTs position as design variable and using the 80 reference WTs of Vestas 80-2MW (same WTs type). Note that inter-space between WTs in the regular configuration (reference layout) is $d_{\mathrm{sw}}=7 \mathrm{D}$ and $d_{\mathrm{per}}=7 \mathrm{D}$, whilst in irregular configuration the separation distance is equal to $d_{\mathrm{sw}}=7 \mathrm{D}$ and $d_{\text {per }}=3.5 D$. Concerning the genetic algorithm used
Table 3 Basic information of four commercial WTs [30, 31]

\begin{tabular}{lcccl}
\hline WTs model & $D$ & $P_{\mathrm{r}}$ & $z_{\mathrm{h}}$ & $V_{\mathrm{r}}$ \\
\hline Vestas66 & 66 & 2 & 60 & 17 \\
Vestas80 & 80 & 2 & 70 & 14.5 \\
Vestas112 & 112 & 3 & 84 & 13 \\
Leanwind164 & 164 & 8 & 110 & 12.5 \\
\hline
\end{tabular}

Table 4 Results of power improvement

\begin{tabular}{llll}
\hline $\begin{array}{l}\text { Layouts com- } \\
\text { parison }\end{array}$ & $\begin{array}{l}\text { Irregular/refer- } \\
\text { ence }\end{array}$ & $\begin{array}{l}\text { Optimized/refer- } \\
\text { ence }\end{array}$ & $\begin{array}{l}\text { Optimized/ } \\
\text { irregular }\end{array}$ \\
\hline $\begin{array}{l}\text { Power improve- } \\
\text { ment (\%) }\end{array}$ & 57,890 & 92,679 & 22,033 \\
\hline
\end{tabular}

to obtain the third configuration, we use the data given in Table 1.

\section{Case 2: Design layout optimization with four types of wind turbine}

According to Eqs. (1), (15) and (19), we remark that rotor diameter of WTs plays a key role in determining wake interactions between WTs, power production and WF cost. This factor is regarded in our study as a crucial design variable to look for optimal WF layout design. Generally speaking when a specific rotor diameter is selected there is a direct impact on inter-space between turbines and number of WTs over a limited WF area. For that reason, our goal behind this case study is to optimize WF layout considering different rotor diameter (type of turbines). Therefore, we consider four commercial WTs V80 (reference turbine), V66, V112 and LW64, characteristics of which are presented in detail in Table 3. The design layout of the three additional WTs studied is constructed and optimized in similar manner as the reference layout V80. It is important to note that the number of WTs during the optimization process has to be defined for each WTs type. The optimization for three additional WTs is conducted under the real conditions of Horns Rev offshore WF.

\section{Optimization results and discussions}

The present study is conducted based on Horns Rev offshore WF characteristics with assumption that all design layouts are optimized under the mean wind speed of $10.6 \mathrm{~m} / \mathrm{s}$ and dominant wind direction of $0^{\circ}$ [32]. Our purpose here is to investigate the impact of some effective inputs variables on power production and cost of WF 
considering the main cases study explained in previous section.

Seeking of how to place WTs inside the limited WF area constitutes a complex task during design process. Indeed, sitting WTs based on classical method cannot always gives a high performance, especially when WTs number is very important.

Therefore, we investigate in the first case under study the influence of three principal configurations on WF power production, efficiency and capacity factor. It should be recalling that 80 WTs of V80-2MW with total power capacity of $160 \mathrm{MW}$ are considered as a reference design layout (Fig. 3a). The power production is evaluated under wake effect taking into account the WTs position as the unique design variable.

Figure 3 illustrates the WF layout of three configurations: the reference layout of Horns Rev offshore WF, irregular and
Table 5 Comparison results of three wind farm design layouts

\begin{tabular}{llll}
\hline WF layouts & Reference & Irregular & Optimized \\
\hline$P_{\mathrm{WF}}(\mathrm{MW})$ & 37.4 & 59.1 & 72.04 \\
$\mathrm{CF}(\%)$ & 23.3 & 36.93 & 45 \\
$\eta(\%)$ & 31.89 & 50.39 & 88 \\
\hline
\end{tabular}

optimized one, and the results are summarized in Table 5. As can be seen, the power production of WTs placed in regular configuration (Fig. 3a) presents a significant power degradation as compared with other configurations. This result can be explained by the fact of high wake interactions between WTs. In such placement, each downstream column of WTs is influenced by multiple wake effect generated among upstream turbines and cannot be avoided while wind direction is equal to $0^{\circ}$. To overcome this issue, we displaced

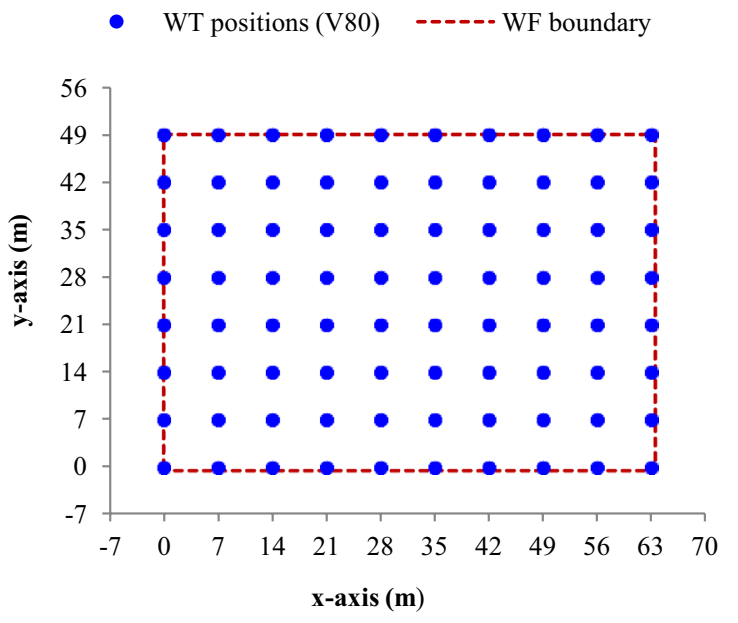

c- Reference layout

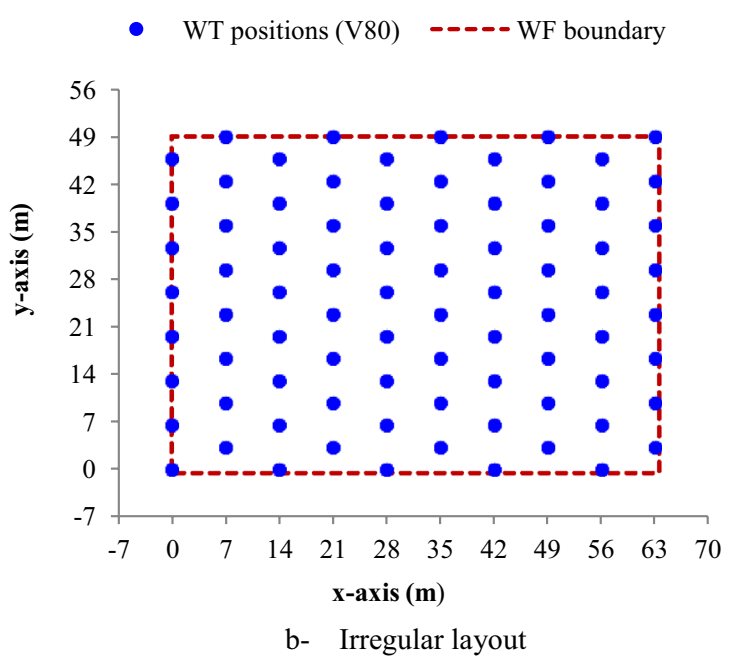

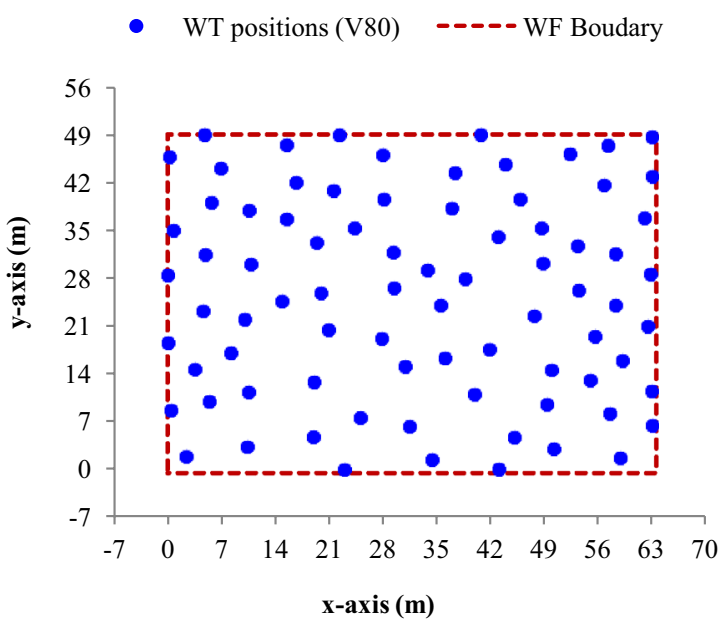

a- Optimized layout

Fig. 3 Illustration of design layouts of hors rev offshore wind farm 


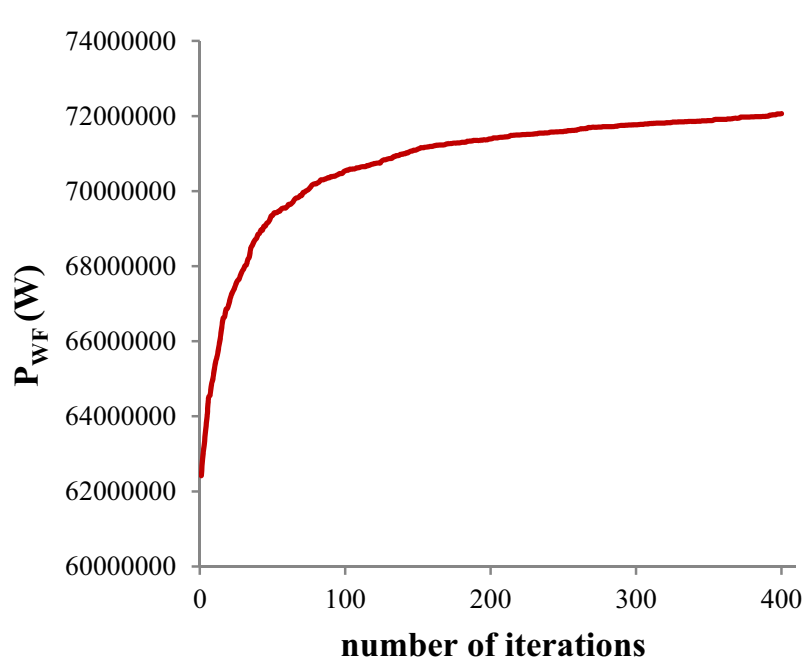

Fig. 4 Convergence history for optimization runs of 400 FOBJ evaluations using GA (optimized layout)
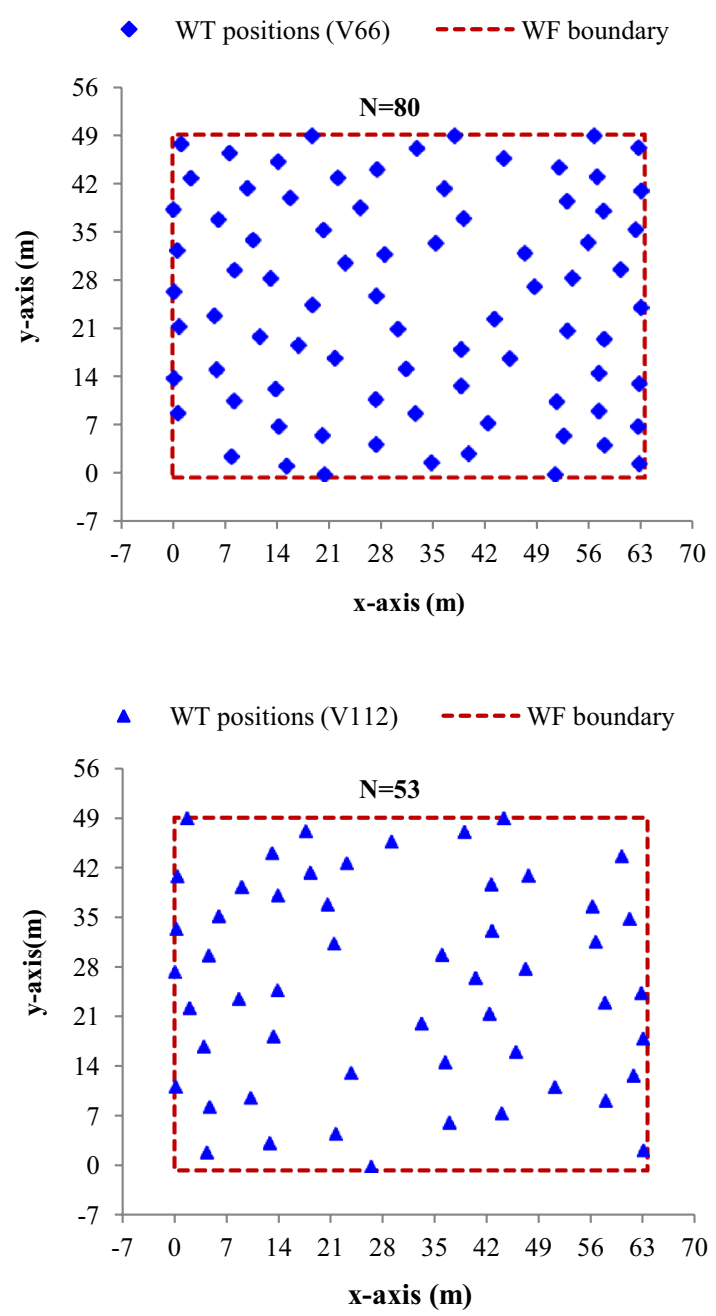

downstream turbines from upstream ones by 3.5 times rotor diameter space to reduce wake interferences (Fig. 3b). It is clearly shown in Table 5 that WF power production considering irregular layout is increased. As shown in Table 4 there is a remarkable improvement about $57.89 \%$ as compared with reference configuration. To explore the possible design layouts of WF and not be limited to regular and irregular design layouts, we demonstrate in Fig. $3 \mathrm{c}$ the effectiveness of optimized layout. The results shown in Table 4 prove that the optimized layout improved the power production about $97 \%$ compared with reference layout and by $22 \%$ compared with irregular one. In addition, a slightly increase in both capacity factor and WF efficiency is noticed (Table 5). It can be concluded from these results that it is crucial for large WF with important number of WTs to use optimization approach to select the optimal WF layout.

Figure 4 presents the history of WFDLO over 400 iterations used in GA. It clearly illustrated that at each iteration

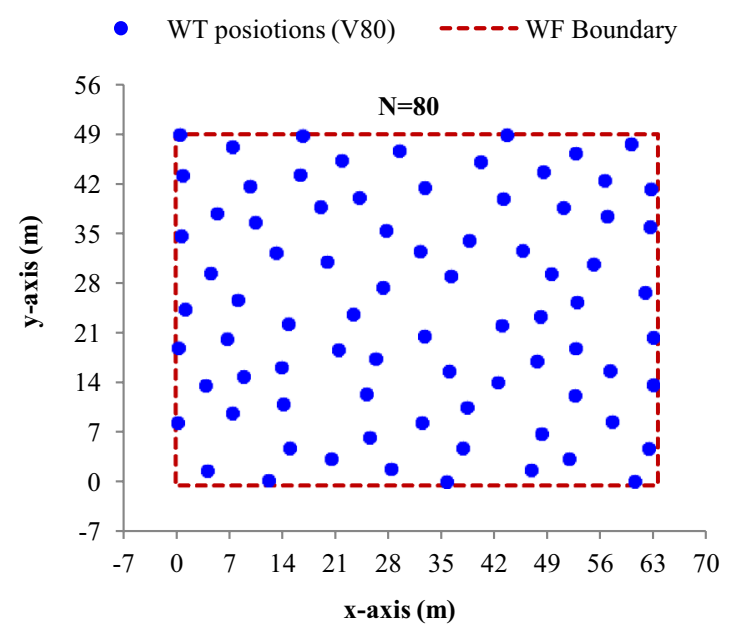

* WT positions (LW164) - - - - - WF boundary

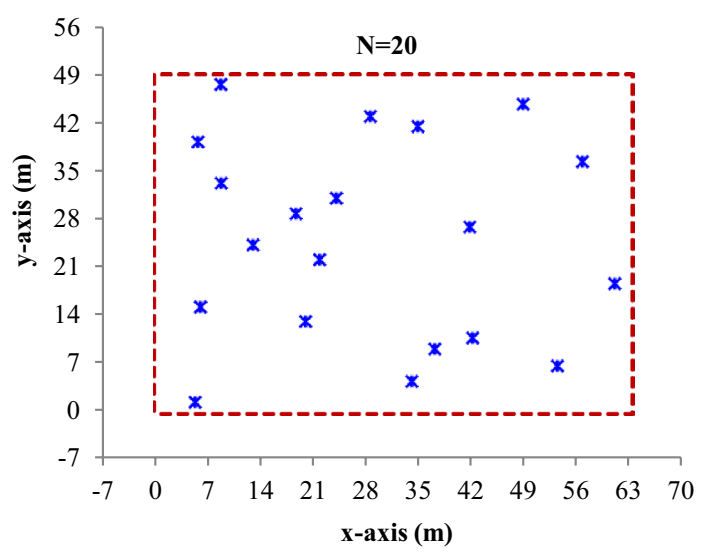

Fig. 5 Design layout optimization of hors rev offshore wind farm using four commercial WTs 
Table 6 Optimization results of WFDL using four wind turbines types

\begin{tabular}{lllll}
\hline WT types & V66 & V80 & V112 & LW164 \\
\hline$P_{\mathrm{WF}}(\mathrm{MW})$ & 47.20 & 71.68 & 107.12 & 94.23 \\
CF $(\%)$ & 30 & 45 & 67 & 59 \\
$\eta(\%)$ & 88 & 87 & 94 & 99.81 \\
\hline
\end{tabular}

Table 7 Cost per unit of power for optimized design layouts

\begin{tabular}{lrrrl}
\hline & \multicolumn{1}{c}{ V66 } & \multicolumn{1}{c}{ V80 } & \multicolumn{1}{c}{ V112 } & LW164 \\
\hline$C_{\mathrm{WF}}(\mathrm{M} \$)$ & 196.48 & 199.04 & 199.22 & 200 \\
$C_{\mathrm{WF}} / P_{\mathrm{WF}}(\$ / \mathrm{W})$ & 4.16 & 2.77 & 1.85 & 2.13 \\
\hline
\end{tabular}

run there is a new generation of WF design layout better than the old one. A gradual improvement in WF power production reflects the effectiveness of proposed algorithm to reach the optimal solution over 400 evaluations of function objective (FOBJ).

The numerical analysis carried out in the second case study aims at finding the optimal positions and choosing the type and number of WTs that give high performances. Figure 4 shows the optimization results of hors rev offshore WF using the four commercial WTs of V80, V66, V112 and LW164. As can be observed from Fig. 5, the design layouts and the number of WTs are strongly depend on WTs size. As much as the WTs size is bigger as the number of installed WTs become fewer. Consequently this provide more free search space that leads to converge easily to optimal design layout. For example, it is clearly seen that WTs are very close when WTs number is important, $N=80$ (for V66 and V80). This can be explained by the fact of limit exploration of search space defined by the constraints of minimum distance between WTs. Whereas in the case of fewer numbers of WTs (V112 and LW164), there is a large possible layout exploration to find optimal placement of WTs inside WF limits.

Further examining the results in Table 6, we can see that the power production and capacity factor increase gradually for WF designed with big rotor diameter except in the case of LW164. Notwithstanding the raises of wake effect with increased rotor diameter size (as can be seen in Eq. 1), wake interferences generated among upstream turbines are weak because of few number of WTs. Accordingly, high power production, capacity factor and efficiency are obtained, respectively, by V112 and 164 as compared with small WTs V66 and V80. The most important observation that can be noted after comparing the performances of these optimized layouts is that designing WF based only on WTs size is not always a good idea. For this reason, the design layout with

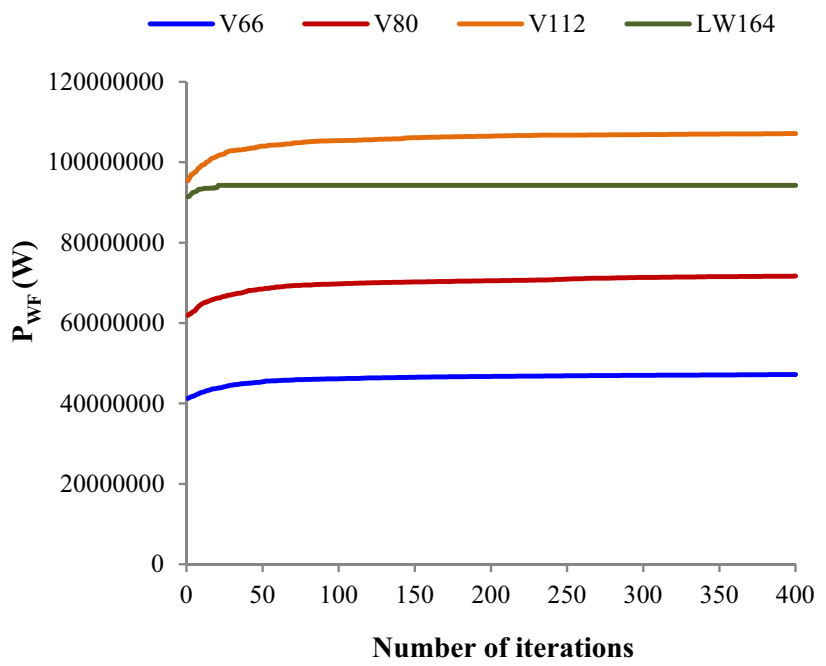

Fig. 6 Convergence histories for four WFDLO

53 WTs of V112 presented best objective performances rather than design layout with LW164.

Note that the performance degradation of WFDL with LW164 can be attributed to the number of WTs preliminarily selected to reach $160 \mathrm{MW}$. Generally speaking the power production of WF increase not only by increasing the size of the rotor diamter of WTs but also with the determined number of WTs. From Table 7, it can be concluded that WF cost and cost per unit of power are also related to the number and size of WTs. The results show that WFDL considering V112 gives the best results in terms of economic objective. In Fig. 6, convergence histories for four WFDLO are depicted.

\section{Conclusion}

In this paper, a new optimization study of realistic WF design layout was performed. The main objective is designing wind farm area under realistic conditions considering different WTs types. In the first step of proposed study, an optimization approach using genetic algorithm with continuous layout representation is conducted to demonstrate and validate the effectiveness and benefit of using such a methodology to seek for optimal WTs placements. Designing the WF layout with four commercial WTs is then investigated and compared in terms of various performance objectives in a second step.

The results show that using an optimization approach instead of designing WF area with traditional rules (regular or irregular layouts) leads to find the optimal design layout with high degree of power production. Otherwise, for a 
given WF area it figured out that a selection of specific WT type plays a key role. Designing an area based only on WT size is not always a good idea. Indeed, the power production, capacity factor, efficiency and cost per unit of power depend strongly on both number and size of WTs. Accordingly, it can be concluded that it is hard to make a choice in terms of power production and WF cost at the same time due to the contradictory aspect of both objectives.

In future studies, it is important to investigate the relationship between WF design layout and the compromise between power production and WF cost. The study will emphasize the need to include other design variables such as using WTs with different hub height. In addition, the study will be conducted under the variability of wind speed and direction of WF.

Open Access This article is distributed under the terms of the Creative Commons Attribution 4.0 International License (http://creativeco mmons.org/licenses/by/4.0/), which permits unrestricted use, distribution, and reproduction in any medium, provided you give appropriate credit to the original author(s) and the source, provide a link to the Creative Commons license, and indicate if changes were made.

\section{References}

1. Wind Europe: wind energy in Europe in 2018, trends and statistics. https://windeurope.org/wpcontent/uploads/files/about wind/statistics/WindEurope-Annual-Statistics-2018.pdf (2018). Accessed 5 Mar 2019

2. Herbert-Acero, J.F., Probst, O., Réthoré, P.E., Larsen, G.C., Castillo-Villar, K.K.: A review of methodological approaches for the design and optimization of wind farms. Energies 7, 6930-7016 (2014)

3. Kim, S.H., Shin, H.K., Joo, Y.C., Kim, K.H.: A study of the wake effects on the wind characteristics and fatigue loads for the turbines in a wind farm. Renew. Energy 74, 536-543 (2015)

4. Diamond, K.E., Crivella, E.J.: Wind turbine wakes, wake effect impacts, and wind leases: using solar access laws as the model for capitalizing on wind rights during the evolution of wind policy standards. Duke Environ. Law Policy Forum 22, 195-244 (2011)

5. Parada, L., Herrera, C., Flores, P., Parada, V.: Wind farm layout optimization using a Gaussian-based wake model. Renew. Energy 107, 531-541 (2017)

6. Khanali, M., Ahmadzadegan, S., Omid, M., Nasab, F.K., Chau, K.W.: Optimizing layout of wind farm turbines using genetic algorithms in Tehran province. Iran. Int. J. Energy Environ. Eng. 9, 399-411 (2018)

7. Wilson, D., Rodrigues, S., Segura, C., Loshchilov, I., Hutter, F., Buenfil, G.L., Peña, S.I.V.: Evolutionary computation for wind farm layout optimization. Renew. Energy 126, 681-691 (2018)

8. Hou, P., Hu, W., Chen, C., Soltani, M., Chen, Z.: Optimization of offshore wind farm layout in restricted zones. Energy 113, 487-496 (2016)

9. González, J.S., Payán, M.B., Santos, J.M.R., González-Longatt, F.: A review and recent developments in the optimal wind-turbine micro-siting problem. Renew. Sustain. Energy Rev. 30, 133-144 (2014)

10. Valverde, P.S., Sarmento, A.J., Alves, M.: Offshore wind farm layout optimization - state of the art. In: The Twenty-Third
International Offshore and Polar Engineering Conference. International Society of Offshore and Polar Engineers (2014)

11. Ulku, I., Alabas-Uslu, C.: A new mathematical programming approach to wind farm layout problem under multiple wake effects. Renew. Energy 136, 1190-1201 (2019)

12. Feng, J., Shen, W.Z.: Optimization of wind farm layout: a refinement method by random search. In: International Conference on Aerodynamics of Offshore Wind Energy Systems and Wakes (ICOWES 2013). Technical University of Denmark (DTU) (2013)

13. Mosetti, G.P.C.D.B., Poloni, C., Diviacco, B.: Optimization of wind turbine positioning in large windfarms by means of a genetic algorithm. J. Wind Eng. Ind. Aerodyn. 51, 105-116 (1994)

14. Grady, S.A., Hussaini, M.Y., Abdullah, M.M.: Placement of wind turbines using genetic algorithms. Renew. Energy 30, 259-270 (2005)

15. Chen, Y., Li, H., Jin, K., Song, Q.: Wind farm layout optimization using genetic algorithm with different hub height wind turbines. Energy Convers. Manag. 70, 56-65 (2013)

16. MirHassani, S.A., Yarahmadi, A.: Wind farm layout optimization under uncertainty. Renew. Energy 107, 288-297 (2017)

17. Mustakerov, I., Borissova, D.: Wind turbines type and number choice using combinatorial optimization. Renew. Energy 35, 1887-1894 (2010)

18. Chowdhury, S., Zhang, J., Messac, A., Castillo, L.: Optimizing the arrangement and the selection of turbines for wind farms subject to varying wind conditions. Renew. Energy 52, 273-282 (2013)

19. Rahbari, O., Vafaeipour, M., Fazelpour, F., Feidt, M., Rosen, M.A.: Towards realistic designs of wind farm layouts: application of a novel placement selector approach. Energy Convers. Manag. 81, 242-254 (2014)

20. Gaumond, M., Réthoré, P.E., Ott, S., Pena, A., Bechmann, A., Hansen, K.S.: Evaluation of the wind direction uncertainty and its impact on wake modeling at the Horns Rev offshore wind farm. Wind Energy 17, 1169-1178 (2014)

21. Méchali, M., Barthelmie, R., Frandsen, S., Jensen, L., Réthoré, P.E.: Wake effects at Horns Rev and their influence on energy production. In: European Wind Energy Conference and Exhibition. Citeseer, vol. 1, pp. 10-20 (2006)

22. Archer, C.L., Vasel-Be-Hagh, A., Yan, C., Wu, S., Pan, Y., Brodie, J.F., Maguire, A.E.: Review and evaluation of wake loss models for wind energy applications. Appl. Energy 226, 1187-1207 (2018)

23. Göçmen, T., Van der Laan, P., Réthoré, P.E., Diaz, A.P., Larsen, G.C., Ott, S.: Wind turbine wake models developed at the technical university of Denmark: a review. Renew. Sustain. Energy Rev. 60, 752-769 (2016)

24. Kermani, N.A., Andersen, S.J., Sørensen, J.N., Shen, W.Z.: Analysis of turbulent wake behind a wind turbine. In: International Conference on Aerodynamics of Offshore Wind Energy Systems and Wakes (ICOWES 2013). Technical University of Denmark (DTU), pp. 53-68 (2013)

25. Shakoor, R., Hassan, M.Y., Raheem, A., Wu, Y.K.: Wake effect modeling: a review of wind farm layout optimization using Jensen' s model. Renew. Sustain. Energy Rev. 58, 1048-1059 (2016)

26. Wang, L., Tan, A.C., Cholette, M., Gu, Y.: Comparison of the effectiveness of analytical wake models for wind farm with constant and variable hub heights. Energy Convers. Manag 124, 189-202 (2016)

27. Jensen, N.O.: A note on wind generator interaction. Technical report Risø-M-2411, Risø National Laboratory, Roskilde (1983)

28. Feng, J., Shen, W.Z.: Wind farm layout optimization in complex terrain: a preliminary study on a Gaussian hill. J. Phys. Conf. Ser. 524, 012146 (2014)

29. Carrillo, C., Montaño, A.O., Cidrás, J., Díaz-Dorado, E.: Review of power curve modelling for wind turbines. Renew. Sustain. Energy Rev. 21, 572-581 (2013) 
30. leanwind.: Logistic efficiencies and naval architecture for wind installations with novel developments summary description of LEANWIND $8 \mathrm{MW}$ reference turbine. http://www.leanwind.eu/ wp-content/uploads/LEANWIND-8-MWturbine_Summary.pdf (2013). Accessed 5 Mar 2019

31. The Wind Power: Wind Energy Market Intelligente. https://www. thewindpower.net/turbines_manufacturers_en.php. Accessed 5 Mar 2019
32. Ceyhan, Ö., Grasso, F.: Investigation of wind turbine rotor concepts for offshore wind farms. J. Phys. Conf. Ser. 524, 012032 (2014)

Publisher's Note Springer Nature remains neutral with regard to jurisdictional claims in published maps and institutional affiliations. 